\title{
Thallium-201 Scintigraphy Was Useful in Diagnosing Ectopic ACTH Syndrome Due to Bronchial Carcinoid
}

\author{
Emina ITOH, IzUmi FUKUdA, Akira SATA, Miho KANAZAWA*, TaKamasa OHNUKI**, \\ NAOMI HIZUKA, KIYOKo KUSAKABE*** AND KAZUE TAKANO \\ Department of Medicine II, Tokyo Women's Medical University, Tokyo 162-8666, Japan \\ * Department of Surgical Pathology, Tokyo Women's Medical University, Tokyo 162-8666, Japan \\ ** Department of Surgery, Chest Institute, Tokyo Women's Medical University, Tokyo 162-8666, Japan \\ *** Department of Radiology, Tokyo Women's Medical University, Tokyo 162-8666, Japan
}

\begin{abstract}
Initial investigations of a 70-year-old woman with clinical Cushing's syndrome, including overnight dexamethasone suppression test, CRH test, and pituitary MRI, suggested the presence of ectopic ACTH production. Thoracic computed tomography $(\mathrm{CT})$ scan revealed a mass measuring $7 \mathrm{~mm}$ in the right lung, but it was thought to be an incidental opacity, leaving the source of ectopic ACTH undetermined for several years. During this period, although the size of the lung opacity did not change remarkably, serum cortisol levels became elevated to $43 \mu \mathrm{g} / \mathrm{dl}$, and the patient's symptoms worsened. Tl-201 SPECT demonstrated intense accumulation in the right lung. The mass was surgically resected using thoracoscopy to investigate it as the focus of ACTH production. Histological and immunohistochemical examination confirmed that the area of intense Tl-201 uptake was an ACTH-producing bronchial carcinoid. Plasma ACTH and cortisol levels decreased immediately after the surgery. In conclusion, this case demonstrated Tl-201 scintigraphy as a useful tool in identifying the location of an ACTH-producing bronchial carcinoid.
\end{abstract}

Key words: Ectopic ACTH syndrome, Bronchial carcinoid, Thallium-201 scintigraphy

(Endocrine Journal 48: 697-702, 2001)

ECTOPIC ACTH secretion accounts for approximately 9-18\% cases of ACTH-dependent Cushing's syndrome [1]. Excluding small cell carcinoma of the lung, most patients present with bronchial, and to a lesser extent, thymic carcinoids. Occult ectopic ACTH syndrome is often clinically indistinguishable from Cushing's disease, as studies of steroid dynamics and pituitary imaging may yield inconclusive or misleading results. Even when the biochemical evidence strongly suggests ectopic ACTH syndrome, it is very difficult to identify the location of the tumor

Received: December 25, 2000

Accepted: September 26, 2001

Correspondence to: Dr. Emina ITOH, Department of Medicine II, Tokyo Women's Medical University, 8-1 Kawada-cho, Shinjuku-ku, Tokyo 162-8666, Japan
$[2,3]$.

We report here a case of a female patient with ectopic ACTH syndrome due to bronchial carcinoid for which Tl-201 scintigraphy was useful in identifying the location of an ectopic ACTH-producing tumor.

\section{Case Report}

In 1994, a 70-year-old woman was admitted to our hospital with a history of hypertension since 1989 and moon face, pigmentation of the skin, acne, and a buffalo hump since 1991. On admission, she was 152 $\mathrm{cm}$ tall and weighed $55 \mathrm{~kg}$. Her blood pressure was $160 / 100 \mathrm{mmHg}$. Biochemical investigations revealed hypokalemia $(3.2 \mathrm{mEq} / \mathrm{L})$ and impaired glucose tol- 
erance after a meal (140-160 mg/dl). Basal plasma levels of ACTH and cortisol were elevated to $134 \mathrm{pg}$ / $\mathrm{ml}$ and $26.5 \mu \mathrm{g} / \mathrm{dl}$, respectively, with loss of the normal diurnal rhythm; both levels failed to be suppressed following overnight dexamethasone $(1,2,8$, $16 \mathrm{mg}$ ) suppression test (Table 1,2$)$. Urinary cortisol excretion level was $475 \mu \mathrm{g} /$ day. From these findings, we diagnosed that she had ACTH-dependent Cushing's syndrome. In support of this diagnosis, plasma ACTH response to $\mathrm{CRH}$ was blunted (Table 3), and dynamic magnetic resonance imaging (MRI) of the pituitary gland showed no evidence of a tumor. These findings strongly suggested ectopic ACTH production and a search for the site of ACTH production ensued. Plasma ACTH increased to $49 \%$ and $75 \%$ of basal after administration of desmopressin and metyrapone, respectively (Table 4). In addition, plasma ACTH decreased to $42 \%$ of basal after octreotide administration $(100 \mu \mathrm{g}, \mathrm{sc})$ (Table 4). Endoscopic examination of the upper gastrointestinal tract, and abdominal CT scan show-

Table 1. Endocrinological findings

\begin{tabular}{|c|c|c|c|c|c|}
\hline \multicolumn{4}{|c|}{ (a) Hormone } & \multicolumn{2}{|c|}{ (normal range) } \\
\hline \multirow[t]{5}{*}{ Serum } & \multicolumn{3}{|c|}{$\mathrm{ACTH}(\mathrm{pg} / \mathrm{ml})$} & 134 & $(10-60)$ \\
\hline & \multicolumn{3}{|c|}{ Cortisol $(\mu \mathrm{g} / \mathrm{dl})$} & 26.5 & $(4.5-24)$ \\
\hline & \multicolumn{3}{|c|}{ DHEA-S $(\mu \mathrm{g} / \mathrm{ml})$} & 2.9 & $(0.6-8.8)$ \\
\hline & \multicolumn{3}{|c|}{ Aldosterone (ng/dl) } & 3.4 & $(2.2-15)$ \\
\hline & \multicolumn{3}{|c|}{$\mathrm{CRH}(\mathrm{pg} / \mathrm{ml})$} & 5.6 & $(3.2-14.7)$ \\
\hline \multirow[t]{3}{*}{ Urine } & \multicolumn{3}{|c|}{ 17-OHCS (mg/day) } & 3.8 & $(1.6-8.8)$ \\
\hline & \multicolumn{3}{|c|}{ 17-KS (mg/day) } & 5.9 & $(2.4-11.3)$ \\
\hline & \multicolumn{3}{|c|}{ Free-cortisol ( $\mu \mathrm{g} /$ day) } & 475 & $(10-150)$ \\
\hline \multicolumn{6}{|c|}{ (b) Circadian fluctuation of serum ACTH and cortisol } \\
\hline \multirow{2}{*}{\multicolumn{2}{|c|}{$\mathrm{ACTH}(\mathrm{pg} / \mathrm{ml})$}} & 0800 & 2000 & & \\
\hline & & 134 & 70 & & \\
\hline \multicolumn{2}{|c|}{ Cortisol $(\mu \mathrm{g} / \mathrm{dl})$} & 26.5 & 24.2 & & \\
\hline
\end{tabular}

ed no evidence of tumor. Thoracic thin slice $(5 \mathrm{~mm})$ CT scan demonstrated a small mass measuring $7 \mathrm{~mm}$ in the right S7 (Fig. 1). However, the mass in right S7 was thought to be an opacity due to old inflammatory changes. At the same time, a brain CT scan revealed a mass measuring $2 \mathrm{~cm}$ in the left posterior lobe that was later diagnosed as a meningioma, which completely resolved following gamma knife therapy. We had initially hypothesized that the meningioma might be the source of ACTH production, but plasma levels of ACTH did not decrease following surgery (Fig. 2). The patient was then treated with metyrapone for hypercortisolemia.

In 1999, the patient presented with hematuria. Cystoscopy revealed a mass measuring $5 \mathrm{~mm}$ in the bladder, which was resected surgically. Pathology indicated a transitional cell carcinoma. ACTH staining of the tumor was performed but yielded a negative result, and plasma ACTH levels remained high. Chest thin slice CT scan was performed yearly to follow the lung mass, but the size of the mass remained unchanged. Since the possibility remained that the mass in right $\mathrm{S} 7$ could be a candidate for the ectopic ACTH-producing tumor, right pulmonary artery sampling was undertaken. The tumor was located in the pulmonary or splanchnic vascular beds, where the veins directly draining the tumor are inaccessible except by trans-left ventricular pulmonary vein catheterization. ACTH levels had to be

Table 2. Overnight dexamethasone suppression test (1994)

\begin{tabular}{lcccc}
\hline & \multicolumn{5}{c}{ Dexamethasone dose (mg) } \\
\cline { 2 - 5 } & 1 & 2 & 8 & 16 \\
\hline ACTH $(\mathrm{pg} / \mathrm{ml})$ & 146 & 100 & 83 & nd \\
Cortisol $(\mu \mathrm{g} / \mathrm{dl})$ & 37.1 & 26.9 & 34.7 & 33.9 \\
\hline
\end{tabular}

nd: not determined

Table 3. Results of preoperative and postoperative CRH test

\begin{tabular}{lcccccccccc}
\hline & \multicolumn{4}{c}{ ACTH $(\mathrm{pg} / \mathrm{ml})$} & & \multicolumn{4}{c}{ Cortisol $(\mu \mathrm{g} / \mathrm{dl})$} \\
\cline { 2 - 4 } \cline { 7 - 10 } & 0 & 30 & 60 & $90(\mathrm{~min})$ & & 0 & 30 & 60 & 90 \\
\hline 1994 & 90 & 104 & 115 & 129 & & 28.0 & 28.1 & 29.0 & 23.0 \\
$00.4^{*}$ & 4.8 & 26.3 & 9.1 & nd & & 1.3 & 2.0 & 1.9 & nd \\
00.9 & 63.2 & 119 & 119 & 83.5 & & 2.9 & 4.9 & 5.2 & 4.8 \\
\hline
\end{tabular}

* One month after surgery

nd: not determined 
Table 4. Desmopressin test (1994)

\begin{tabular}{|c|c|c|c|c|c|c|c|}
\hline \multicolumn{4}{|c|}{ ACTH $(\mathrm{pg} / \mathrm{ml})$} & \multicolumn{4}{|c|}{ Cortisol $(\mu \mathrm{g} / \mathrm{dl})$} \\
\hline 0 & 30 & 60 & $90(\mathrm{~min})$ & 0 & 30 & 60 & 90 \\
\hline 86 & 118 & 128 & 126 & 27.9 & 31.5 & 31.5 & 27.6 \\
\hline
\end{tabular}

Metyrapone test (1994)

\begin{tabular}{lccccc}
\hline & \multicolumn{5}{c}{ Time $(\mathrm{hr})$} \\
\cline { 2 - 6 } & 0 & 2 & 4 & 6 & 8 \\
\hline ACTH $(\mathrm{pg} / \mathrm{ml})$ & 100 & 209 & 243 & 275 & 165 \\
Cortisol $(\mu \mathrm{g} / \mathrm{dl})$ & 29.7 & 7.3 & 7.5 & 9.7 & nd \\
11-deoxycortisol $(\mathrm{ng} / \mathrm{ml})$ & 6.9 & 205 & 280 & 167 & nd \\
\hline
\end{tabular}

Octreotide test (1998)

\begin{tabular}{lcccccccc}
\hline & \multicolumn{10}{c}{ Time $(\mathrm{hr})$} \\
\cline { 2 - 10 } & 0 & 2 & 3 & 4 & 5 & 6 & 7 & 8 \\
\hline ACTH $(\mathrm{pg} / \mathrm{ml})$ & 877 & 587 & 646 & 689 & 506 & 554 & 439 & 503 \\
Cortisol $(\mu \mathrm{g} / \mathrm{dl})$ & 16.4 & 17.2 & 19.9 & 24.8 & 34.2 & 37.4 & 17.9 & 16.2
\end{tabular}

nd: not determined

This case was reported in Japanese Haigan journal in April 2001.

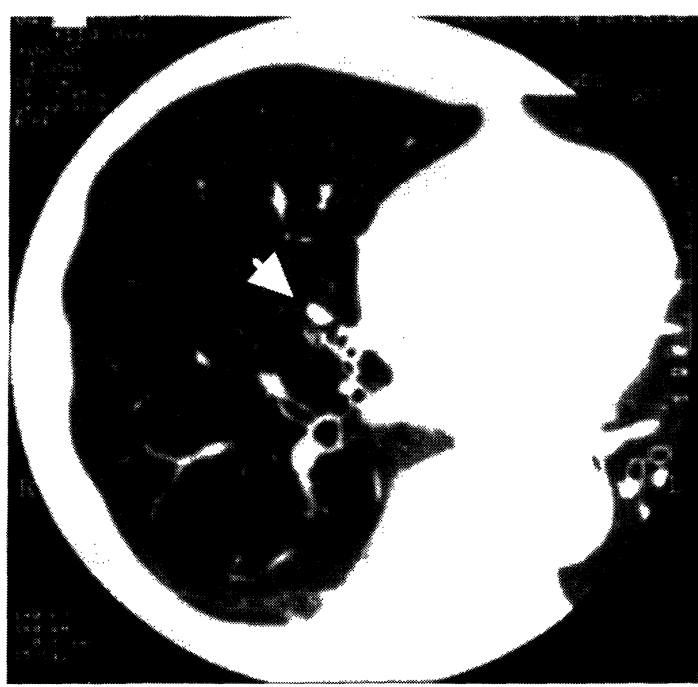

Fig. 1. Chest CT. Arrows indicate a tumor.

measured in the wedged pulmonary artery instead of the pulmonary vein. The results of this procedure revealed no differences between plasma ACTH levels from the pulmonary artery $(1219 \mathrm{pg} / \mathrm{ml})$, and peripheral artery $(1375 \mathrm{pg} / \mathrm{ml})$. Thallium perfusion scintigraphy following this procedure revealed uptake in the right lower lung field, again furthering suspicion that this mass was the source of ectopic ACTH (Fig. 3).

In 2000 , the patient presented with overt diabetes mellitus, and her hypertension became uncontrolled despite metyrapone treatment. Basal plasma ACTH and cortisol levels were elevated at $1000-1500 \mathrm{pg} / \mathrm{ml}$ and $40-45 \mu \mathrm{g} / \mathrm{dl}$, respectively. Endoscopic examination of the lower gastrointestinal tract was normal. Again, thoracic CT scan demonstrated the mass measuring $7 \mathrm{~mm}$ in the right lower lobe (S7) and it was thought to be the same lesion that was demonstrated by Tl-201 SPECT. Therefore, it was considered to be a candidate for the ACTH-producing tumor, and the tumor was surgically resected by thoracoscopy. During the surgical procedure, the tumor was found to be in the right middle lobe. This anomaly of the right middle lobe was what was thought to be in S7 on CT scan before surgery. The tumor was round, $8 \times 5 \mathrm{~mm}$ and yellow. Histological examination of the tumor demonstrated a typical carcinoid morphology and immunohistochemical staining indicated that the majority of tumor cells exhibited positive cytoplasmic staining for ACTH (Fig. 4), chromogranin A, synaptophysin and neuron-specific enolase (NSE), thereby confirming a di- 


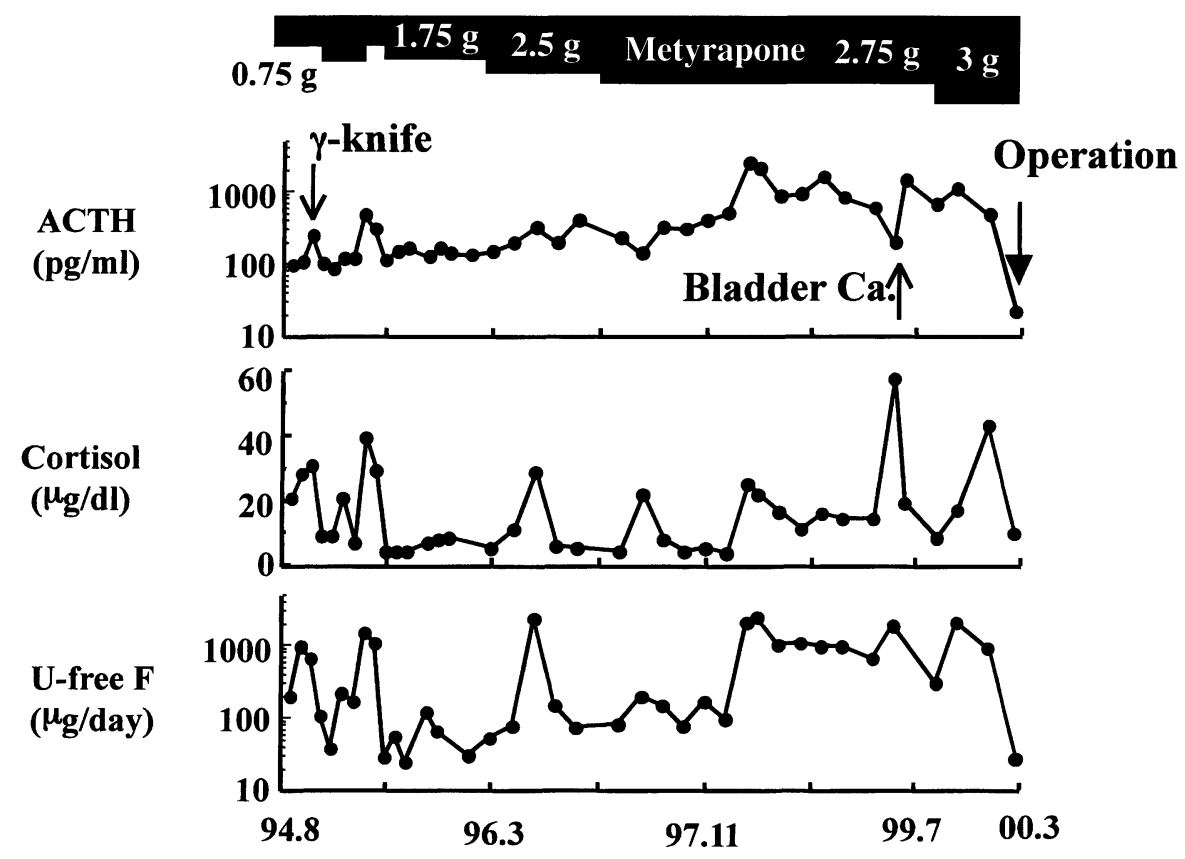

Fig. 2. Clinical course and ACTH, cortisol and urine cortisol levels of the case. Plasma levels of ACTH did not decrease following gamma knife surgery and resection of bladder carcinoma. After the surgery of thoracic tumor, basal ACTH and cortisol levels decreased immediately.

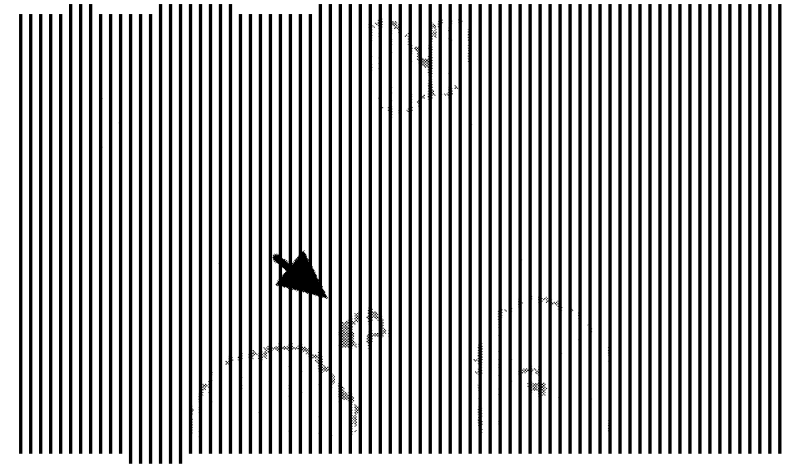

Fig. 3. Thallium perfusion scintigraphy of the lung. Arrow indicates increased uptake in the right lower lung field.

agnosis of an ACTH-secreting carcinoid tumor. In addition to the $8 \times 5 \mathrm{~mm}$ mass, there was another tumor measuring $3 \mathrm{~mm}$. It did not stain for ACTH but was positive for chromogranin $A$ and NSE. Basal ACTH and cortisol levels immediately decreased 30 minutes after surgery to $16 \mathrm{pg} / \mathrm{ml}$ and $3.1 \mu \mathrm{g} / \mathrm{dl}$, respectively (Fig. 2). Hyperglycemia and hypertension were improved. Two weeks after the surgery, plasma ACTH and cortisol were suppressed

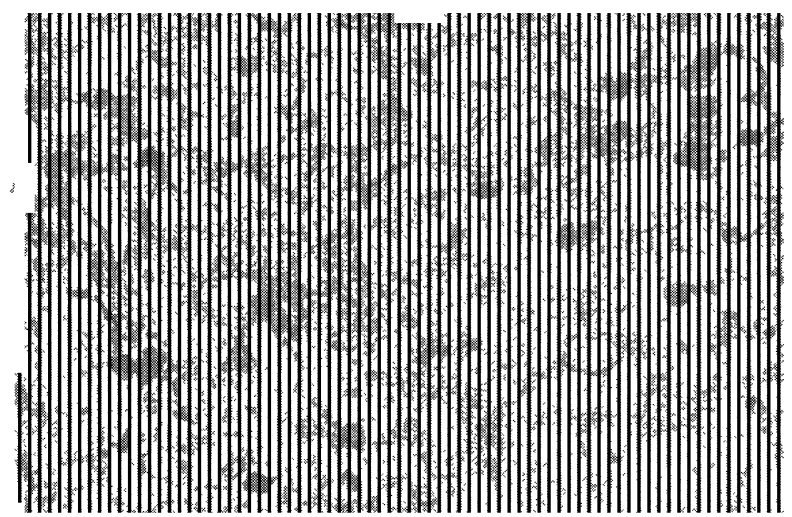

Fig. 4. Immunostaining of the tumor for ACTH. Tumor cells display a cytoplasmic staining for ACTH (brown). $(\times 200)$

normally by dexamethasone $(1 \mathrm{mg})$ suppression test. ACTH and cortisol responsiveness to CRH recovered eight months after the surgery. Replacement therapy with hydrocortisone was discontinued and the patient is doing well. 


\section{Methods}

\section{Clinical examinations}

Dexamethasone suppression tests $(1,2,8,16 \mathrm{mg})$ were performed by overnight method.

CRH (hCRH Mitsubishi Injection) or desmopressin (Kyowa Hakko) was injected at 9:00 am as an intravenous bolus of $100 \mu \mathrm{g}$ or $5 \mu \mathrm{g}$, respectively. A $50 \%$ increase in plasma $\mathrm{ACTH}$ or a $20 \%$ increase in plasma cortisol over basal levels was regarded as responsive [4].

Following an oral administration of $1.5 \mathrm{~g}$ metyrapone, plasma ACTH, cortisol, and 11-deoxycortisol were measured every two hours until 8 hours after administration. Following the subcutaneous injection of $100 \mu \mathrm{g}$ octreotide (Novartis), plasma ACTH and cortisol were measured every hour until 8 hours after administration.

\section{Histological examination}

The tumor section was immunostained with a rabbit polyclonal anti-human ACTH antibody (Dako, Glostrup, Denmark) at a dilution of $1: 250$. Immunoreaction product deposits were visualized by the avidin-biotin immunoperoxidase complex (ABC) procedure. Reaction control samples of sections were prepared with an incidental procedure except for the reaction with primary antibodies.

\section{Discussion}

Occult ectopic ACTH syndrome is defined as CRH and/or ACTH-dependent hypercortisolemia of nonpituitary origin of more than 4-6 months duration without the emergence of an obvious source [1]. There are two major problems with a patient who has Cushing's syndrome due to occult ectopic ACTH production. First, it is often clinically indistinguishable from Cushing's disease, as biochemical studies of steroid dynamics and pituitary imaging may yield inconclusive or misleading results. Secondly, it is difficult to determine the source of ectopic ACTH production because of the variable location and small size of the tumors $[5,6]$.

In this case, a small intrapulmonary lesion on CT was identified in 1994, but it was thought to be an incidental opacity due to old inflammatory changes. The important point in this case was that the Tl-201 scintigraphy images demonstrated marked accumulation of radioactivity in the area of the lesion of right lung corresponding to the location of the tumor on CT. Tonami et al. reported a case of carcinoid tumor of the thymus in which Tl-201 SPECT demonstrated intense accumulation in the tumor [7]. They speculated that intense uptake by multiple, solid small cell nests of closely packed cells in the viable part of the tumor were responsible for $\mathrm{Tl}$ accumulation and prolonged retention. Ando et al. reported that a small fraction of thallium taken up by tumor cells was localized in the nuclear mitochondrial and microsomal fractions of tumor tissue [8]. Although Tl-201 scintigraphy is not specific for carcinoid or for ACTH-producing tumors, Elgazzar et al. reported that Tl-201 uptake reflects the viability and metabolic activity of the tumor cells [9]. In our case, the tumor was an ACTH-producing bronchial carcinoid that might be viable and have high metabolic activity. It was invaluable to this case that Tl-201 SPECT identified the location of the tumor.

Recently, somatostatin receptor scintigraphy (SRS) has been proposed for the localization of ectopic ACTH-producing tumors [10, 11]. At present, SRS has not been approved in Japan. However, Tabarin et al. reported that the sensitivity of SRS for the detection of bronchial carcinoids was lower than that of thin-section CT scanning [12]. It has also been shown that the positivity of SRS relies not on tumor size but on the density or subtypes of the receptors expressed [12, 13].

In conclusion, this case suggests that thallium perfusion scintigraphy might be useful when it is difficult to identify the source of ectopic ACTH production.

\section{References}

1. Wajchenberg BL, Mendonca BB, Liberman B, Pereira MA, Carneiro PC, Wakamatsu A, Kirschner
MA (1994) Ectopic adrenocorticotropic hormone syndrome. Endocr Rev 15: 752-787. 
2. Vincent JM, Trainer PJ, Renzek RH, Marcus AJ, Dacie JE, Armstrong P, Besser GM (1993) The radiological investigation of occult ectopic ACTH-dependent Cushing's syndrome. Clinical Radiology 48: 11-17.

3. Doppman JL, Nieman L, Miller DL, Pass HI, Chang R, Cutler GB Jr, Schaaf M, Chrousos GP, Norton JA, Ziessman HA (1989) Ectopic adrenocorticotoropic hormone syndrome: localization studies in 28 patients. Radiology 172: 115-124.

4. Kaye TB, Crapo L (1990) Cushing's syndrome: an update on diagnostic tests. Ann Intern Med 112: 435444.

5. Kumagai M, Suzuki S, Yumita W, Minemura K, Ikeo Y, Inagaki T, Hiramatsu K, Aizawa T, Hashizume K (2001) An ectopic ACTH-producing carcinoid tumor localized by the measurement of ACTH in the bronchial lavage. Endocr $J$ 48: 363-367.

6. Ohta K, Shichiri M, Kameya T, Matsubara O, Imai T, Marumo F, Hirata Y (2000) Thymic hyperplasia as a source of ectopic ACTH production. Endocr J 47: 487-492.

7. Tonami N, Yokoyama K, Nonomura A, Taki J, Hisada K, Watanabe Y, Takashima T (1994) Intense accumulation of $201 \mathrm{Tl}$ in carcinoid tumor of the thymus. Clin Nucl Med 19: 408-412.

8. Ando A, Ando I, Katayama M, Sasada S, Hiraki T, Mori H, Tonami N, Hisada K (1987) Biodistribution of T1-201 in tumor bearing animals and inflammatory lesions induced animals. Eur J Nucl Med 12: 567-572.

9. Elgazzar AH, Fernandez-Ulloa M, Silberstein EB (1993) Tl-201 as a tumor localizing agent: current status and future considerations. Nucl Med Commun 14: 96-103.

10. Tsagarakis S, Giannakenast C, Vassilakost PJ (1995) Successful localization of an occult ACTH-secreting bronchial carcinoid tumor with 111 indium-DTPA labelled octoreotide. Clin Endocrinol 43: 763-767.

11. Christin-Maitre S, Chabbert-Buffet N, Mure A, Boukhris R, Bouchard P (1996) Use of somatostatin analog for localization and treatment of $\mathrm{ACTH}$ secreting bronchial carcinoid tumor. Chest 109: 845846.

12. Tabarin A, Valli N, Chanson P, Bachelot $\mathrm{Y}$, Rohmer V, Bex-Bachellerie V, Catargi B, Roger P, Laurent F (1999) Usefulness of somatostatin receptor scintigraphy in patients with occult ectopic adrenocorticotropin syndrome. J Clin Endocrinol Metab 84: 11931202.

13. Krenning EP, Kwekkeboom DJ, Bakker WH, Breeman WA, Kooij PP, Oei HY, van Hagen M, Postema PT, de Jong M, Reubi JC (1993) Somatostatin receptor scintigraphy (111In-DTPA-d-Phel)and (123I-Tyr 3)-octreotide: the Rotterdam experience with more than 1000 patients. Eur J Nucl Med 20: 716-731. 